\title{
ÍNDICE DE SUSTENTABILIDADE DE BACIAS HIDROGRÁFICAS: ESTUDO DE CASO DAS SUB-BACIAS DO BATATÃ E MARACANÃ, SÃO LUÍS/MA
}

\author{
SUSTAINABILITY INDEX OF WATERSHEDS: CASE STUDY OF THE \\ SUB-BASINS OF BATATÃ AND MARACANÃ, SÃO LUÍS/MA
}

\section{Leonardo Silva Soares ${ }^{1}$ \\ Antônio Carlos Leal de Castro² Marcelo Henrique Lopes Silva ${ }^{3}$ Edson Vicente da Silva ${ }^{4}$}

\begin{abstract}
1 Curso de Engenharia de Pesca, Universidade Federal do Maranhão. E-mail: leonardoufma@yahoo.com.br 2 Departamento de Oceanografia, Universidade Federal do Maranhão. E-mail: alec@ufma.br 3 Curso de Ciências Humanas, Universidade Federal do Maranhão. E-mail: arkleymbandeira@gmail.com

4 Doutor e Docente do Curso de Geografia, Universidade Federal do Ceará. E-mail: cacauceara@gmail.com
\end{abstract}

RESUMO: Este artigo objetiva avaliar a aplicação do Índice de Sustentabilidade de Bacias Hidrográficas como subsídio para formulação de políticas públicas de conservação para as sub-bacias hidrográficas do Batatã e Maracanã, São Luís, Maranhão. O índice é composto por quatro dimensões: Ambiental, Qualidade de Água, Socioeconômica e Político-Institucional. As dimensões são compostas por indicadores e estes, em alguns casos, por variáveis básicas, obtidas em campo e laboratório. Comparativamente, os valores obtidos para a sub-bacia do Batatã foram superiores em relação à sub-bacia do Maracanã em todas as dimensões analisadas.

Palavras-chave: Gestão Ambiental. Índices de Sustentabilidade. Políticas Públicas. Conservação.

\begin{abstract}
This article aims to evaluate the application of the Hydrographic Basin Sustainability Index as a subsidy for the formulation of public conservation policies for the hydrographic sub-basins of Batatã and Maracanã, São Luís, Maranhão. The index is composed of four dimensions: Environmental, Water Quality, Socioeconomic and Political-Institutional. The dimensions are composed of indicators and these, in some cases, by basic variables, obtained in the field and laboratory. Comparatively, the values obtained for the Batatã sub-basin were higher in relation to the Maracanã sub-basin in all dimensions analyzed.
\end{abstract}

Keywords: Environmental Management. Sustainability Indices. Public policy. Conservation

Sumário: Introdução - 1 As Sub-Bacias Hidrográficas dos Rios Batatã e Maracanã - 2 - As Dimensões Ambiental e Socioeconômica das SBH do Batatã e Maracanã - 3 Análise Integrada do Índice de Sustentabilidade de Bacias Hidrográficas Considerações Finais - Referências

\section{INTRODUÇÃO}

As bacias hidrográficas são delimitadas a partir de uma área drenada por um rio principal e seus tributários, sendo limitadas pelos divisores de água. Estas áreas são consideradas unidades naturais de planejamento, podendo, também, serem usadas como unidades de manejo, uma vez que nelas se desenvolve todas as atividades de uso e ocupação do solo. A qualidade ambiental de uma bacia hidrográfica pode ser avaliada, por um conjunto de indicadores específicos, derivado de um modelo de informação que represente a realidade da bacia. Construir indicadores de sustentabilidade é algo complexo, uma vez que eles devem refletir a relação da sociedade com o meio ambiente numa perspectiva ampla, considerando todos os fatores envolvidos no processo.

Os indicadores podem ser utilizados como ferramenta para subsidiar o planejamento ambiental, pois 
ajudam a determinar as alterações no ambiente, selecionando medidas chaves, que podem ser físicas, químicas, biológicas ou socioeconômicas, e que oferecem informações úteis à compreensão do ambiente como um todo. Estudiosos relatam que as bacias urbanas necessitam ser planejadas considerando os impactos do uso e ocupação do solo sobre os recursos hídricos. Contudo, a falta de planejamento adequado e as irregularidades na ocupação descontrolada tornam esta tarefa bastante difícil. Assim, devem ser reconhecidos indicadores apropriados para direcionar a sustentabilidade dos centros urbanos e seu entorno.

A busca por instrumentos que reduzam a incerteza na tomada de decisão, como os índices e indicadores, configura uma importante ferramenta para os gestores, pois ocorrem situações em que o tomador de decisão tem pouco ou nenhum conhecimento ou informação para atribuir probabilidades a cada estado da natureza ou a cada evento futuro. Neste contexto de ausência de planejamento, de utilização de critérios para tomada de decisão e de depreciação dos recursos naturais estão inseridas as bacias hidrográficas localizadas no município de São Luís/MA. Nestas áreas, historicamente observou-se o rápido crescimento demográfico e a multiplicidade de atividades humanas que exploram e consomem os recursos naturais e, simultaneamente, produzem diversas quantidades de resíduos, poluentes e interferências no ambiente natural.

Dentre as principais bacias hidrográficas do município de São Luís, destaca-se a do Bacanga que apresenta grande complexidade e relevância ambiental e é possível observar que o crescimento progressivo da população na bacia, aliada ao aumento do uso e ocupação do solo que se processa de forma desordenada, vem contribuindo para o aumento da compactação do solo, assoreamento e contaminação dos corpos de água da bacia. Devido estes aspectos é possível afirmar que há necessidade de se planejar o crescimento da bacia, haja vista ser considerada uma das principais contribuintes para o abastecimento de água da cidade de São Luís.

Assim, este trabalho propõe em avaliar e aplicar o Índice de Sustentabilidade de Bacias Hidrográficas (ISBH) nas Sub-Bacias Hidrográficas $(\mathrm{SBH})$ do Batatã e Maracanã que integram a bacia do Bacanga. A escolha destas SBH sejustifica pela importância socioeconômica e ambiental destas áreas. A primeira (Batatã) integra grande parte da área do Parque Estadual do Bacanga e da Zona de Reserva Florestal do Sacavém. Nesta SBH está situado o reservatório Batatã que é responsável pela captação de água que abastece parte da Cidade de São Luís. Enquanto na SBH do Maracanã, estão localizadas as principais nascentes do rio Bacanga e grande parte da Área de Proteção Ambiental do Maracanã.

Diante do exposto, impõem-se como necessidade prioritária investigar cientificamente procedimentos e indicadores para a análise do processo de planejamento das $\mathrm{SBH}$, no sentido de propiciar a elaboração de instrumentos técnico-científicos para sua medição, auxiliando as tomadas de decisões e a gestão sustentável da atividade na região do Maracanã e Batatã.

As informações geradas com a pesquisa poderão contribuir para o estabelecimento de medidas de preservação, conservação e recuperação das SBH estudadas. A utilização do ISBH como uma ferramenta de gestão almeja sustentar o processo de tomada de decisão, por meio da avaliação da informação, convertendoa numa série de medidas úteis e significativas, subsidiando uma análise de tendências de agravamento de conflitos e reduzindo as probabilidades de se adotar decisões equivocadas, principalmente na formulação de políticas públicas.

Desta forma, este trabalho tem como objetivo avaliar a aplicação do ISBH nas SBH do Batatã e Maracanã, São Luís/MA, como instrumento de planejamento ambiental e socioeconômico de auxílio à formulação de políticas públicas no sentido de promover a sustentabilidade da região.

\section{AS SUB-BACIAS HIDROGRÁFICAS DOS RIOS BATATÃ E MARACANÃ}

São Luís é um dos quatro municípios que fazem parte da Ilha Upaon-Açu (denominação dada pelos índios tupinambás significando Ilha Grande), está localizada entre as coordenadas de 02 24'09" e 0246'13"

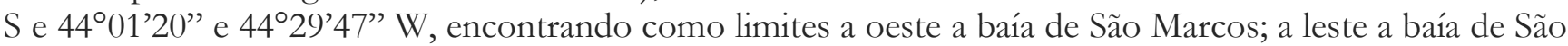
José; ao sul o Estreito dos Mosquitos e ao norte o Oceano Atlântico. Na ilha, existem também, os municípios de São José de Ribamar, Paço do Lumiar e Raposa (COELHO, 2006). 
No espaço da ilha, a bacia do rio Bacanga, com uma superfície da ordem de 11.030,00 ha, ocupa a porção Noroeste, fazendo parte do município de São Luís com localização definida pelas coordenadas

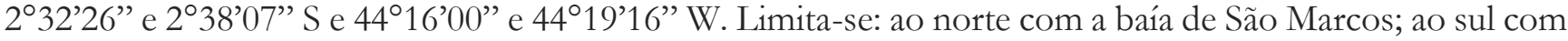
o tabuleiro central da ilha na região do Tirirical; a leste com o divisor de águas que separa as bacias dos rios Anil, Paciência e Tibiri e a oeste, pelo divisor de águas que a separa das bacias do Bacanga da bacia Litorânea oeste (banhada pelas águas da baía de São Marcos).

A bacia do Bacanga correspondente a 12,33\% do território no município de São Luís, com perímetro de 44,2 km e curso d'água principal com 19 km de extensão. É composta por 12 (doze) SBH, onde encontram-se aproximadamente 64.000 domicílios, o que corresponde a uma estimativa populacional de aproximadamente 256.000 habitantes, distribuídos por cerca de sessentas bairros, entre conjuntos habitacionais e ocupações irregulares. Estes seus limites estão inseridos trechos de três Unidades de Conservação (UC), sendo elas: a APA de Maracanã, APA de Upaon-Açu/Miritiba/Alto Preguiça e o Parque Estadual do Bacanga. Possui também em seu território a Reserva Florestal do Sacavem, além da presença do Distrito Industrial de São Luís (CASTRO, 2008).

Dentre as 12 (doze) SBH destacam-se as do Batatã e Maracanã (Figura 1) que estão situadas nas cabeceiras do sistema Bacanga, possuindo atributos ambientais relevantes caracterizados por ecossistemas remanescentes, disponibilidade de recursos hídricos, potencial turístico e possibilidade da implementação de políticas públicas voltadas para o planejamento e gestão ambiental.

Figura 1 - Localização da BH do Bacanga, destaque SBH do Batatã e Maracanã

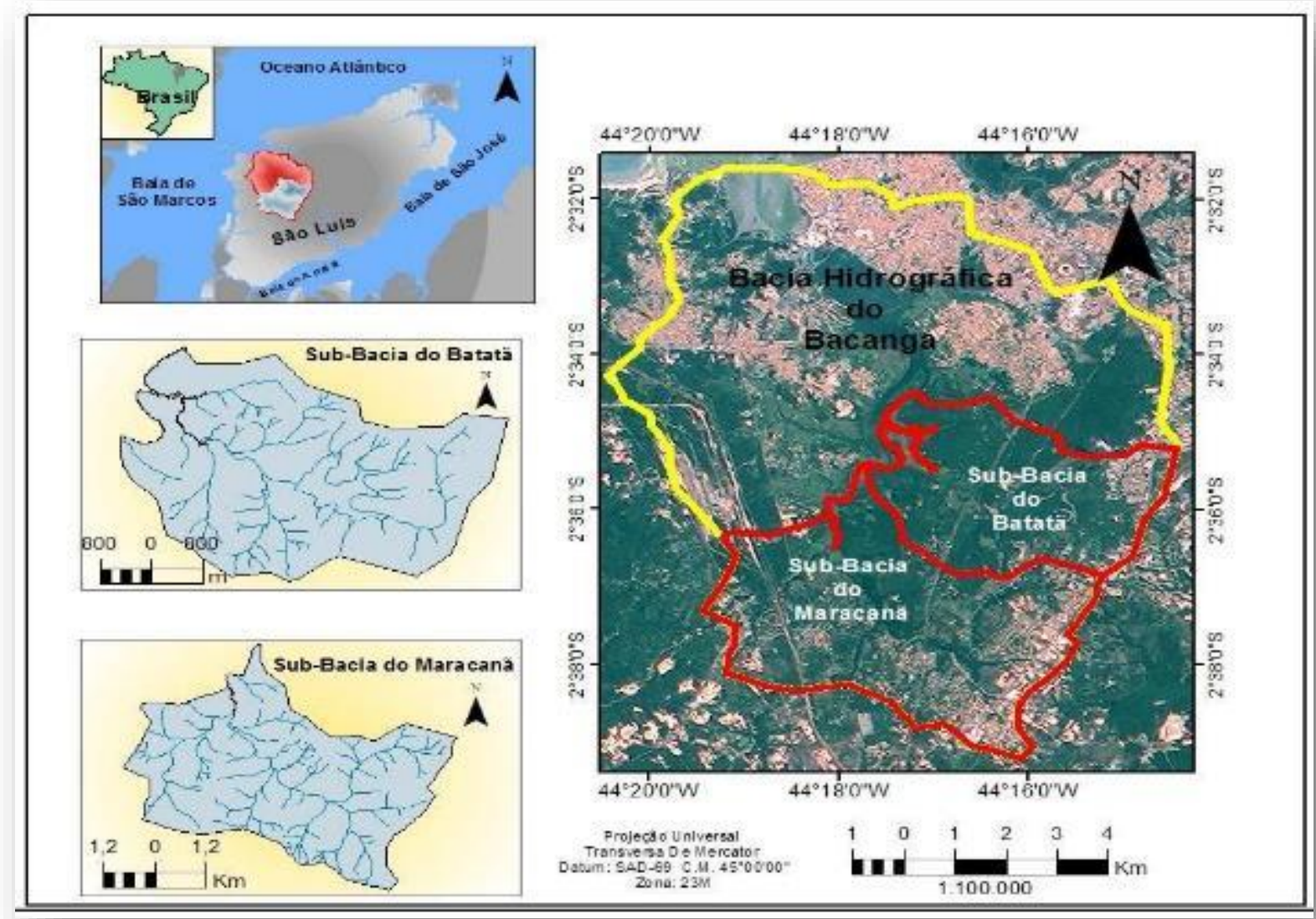

Fonte: Autores (2018). 
A SBH do Batatã possui uma área de 1.732 hectares e está compreendida entre as coordenadas

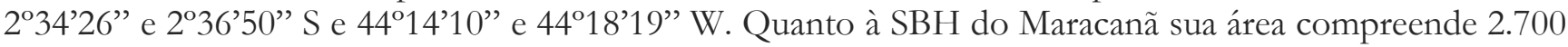

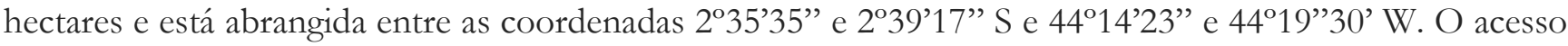
rodoviário principal se faz pela BR-135, após atravessar o Estreito dos Mosquitos, canal que liga a ilha ao continente maranhense (PEREIRA, 2006). Para subsidiar a aplicação do ISBH foram utilizados os seguintes materiais:

(1) Cartas Planialtimétricas DSG (1980), folhas 30, 31 e 38 na escala 1:10.000: Obtidas no ZEE-MA (2003);

(2) Aerofoto do Serviço Aéreo do Cruzeiro do Sul da bacia do Bacanga, capturada em 1976 (Diretoria de Hidrografia e Navegação Marinha - 1976);

(3) Fusão de Imagem SPOT, QUICKBIRD e IKONOS II capturadas em 2008;

(4) Mapa pedológico de São Luís, na escala 1:100.000 (MARANHÃO, 2002);

(5) Bases digitais (Curvas de nível, hidrografia, arruamento, unidades de paisagem, UC e zonas de reserva florestal, áreas de preservação permanente, limites de bacias hidrográficas, todas em formato SHP) e;

(6) Dados históricos de pluviometria de duas estações climatológicas de São Luís (UEMA e INMET) no período de 1993 a 2008.

O ISBH apresentado neste trabalho foi desenvolvido por Isaias (2008) e integra indicadores nas seguintes dimensões: Ambiental, de Qualidade da Água, Socioeconômico e Político-Institucional. Segundo este autor a dimensão ambiental abrange, conceitualmente e, de certo modo também na prática, a dimensão de qualidade da água. No entanto, sob a justificativa de conferir maior peso aos fatores relacionados à qualidade da água, uma vez que se trata de um índice de sustentabilidade de bacias hidrográficas, optou-se por se elevar ao status de dimensão o conjunto de indicadores de qualidade da água.

Para o cálculo ISBH considera-se o mesmo peso entre as dimensões envolvidas no índice. Cada indicador representante das dimensões envolvidas na fórmula varia na escala de 0 a 1 , onde 0 indica à pior situação do indicador e 1 a melhor. A fórmula (01) representa o valor adimensional do ISBH.

$$
\mathrm{ISBH}=\{\mathrm{DA}(0-1)+\mathrm{DQ}(0-1)+\mathrm{DS}(0-1)+\mathrm{DP}(0-1)\} / 4(01),
$$

Onde:

(DA) = Indicador da Dimensão Ambiental;

$(\mathrm{DQ})=$ Indicador da Dimensão de Qualidade da Água;

(DS) = Indicador da Dimensão Socioeconômico; e

(DP) = Indicador da Dimensão Político Institucional.

A composição e o cálculo do ISBH são apresentados nas partes subsequentes. No entanto, a Tabela 1 resume as dimensões, indicadores e variáveis básicas que constituem o ISBH. O fluxograma apresentado na Figura 2, objetiva facilitar a visualização do ISBH, destacando as dimensões, indicadores e variáveis básicas que o compõem. 
Tabela 1 - Resumo do cálculo do ISBH

\begin{tabular}{|c|c|c|}
\hline İndice & Dimensões & $\begin{array}{c}\text { Indicadores e Variáveis } \\
\text { Básicas }\end{array}$ \\
\hline \multirow{4}{*}{$\begin{array}{l}\text { Índice de Sustentabilidade de } \\
\text { Bacias Hidrográficas } \\
\text { ISBH }=\{\mathrm{DA}(0-1)+\mathrm{DQ}(0-1) \\
+\mathrm{DS}(0-1)+\mathrm{DP}(0-1)\} / 4\end{array}$} & $\begin{array}{c}\text { Dimensäo Ambiental } \\
\mathrm{DA}=\{\mathrm{CV}(0-1)+\mathrm{RE}(0-1)+ \\
\mathrm{De}(0-1)+\mathrm{Al}(0-1)\} / 4\end{array}$ & $\begin{array}{l}\text { CV = Indicador de Cobertura } \\
\text { Vegetal; RE = Indicador de } \\
\text { Risco de Erosão; De = } \\
\text { Indicador Densidade de } \\
\text { Estradas; Al = Indicador de } \\
\text { Área Impermeabilizada. }\end{array}$ \\
\hline & $\begin{array}{c}\text { Dimensão de Qualidade de } \\
\text { Água } \\
\mathrm{DQ}=\{\operatorname{IQA}(0-1)+\mathrm{T}(0-1)\} / 2\end{array}$ & $\begin{array}{l}\text { IQA = Indicador referente ao } \\
\text { İndice de Qualidade da Água; } \\
T=\text { Indicador de Turbidez. }\end{array}$ \\
\hline & $\begin{array}{c}\text { Dimensão socioeconômica } \\
\mathrm{DS}=\{\mathrm{R}(0-1)+\mathrm{Ed}(0-1)+ \\
\mathrm{SP}(0-1)\}\end{array}$ & $\begin{array}{l}\mathrm{R}=\text { Indicador de Renda; } \mathrm{Ed}= \\
\text { Indicador de Educação; SP = } \\
\text { Saúde Pública. }\end{array}$ \\
\hline & $\begin{array}{c}\text { Dimensão Politico } \\
\text { Institucional } \\
\begin{array}{c}\mathrm{DP}=\{\mathrm{TUE}(0-1)+\operatorname{IAPP}(0-1) \\
+\mathrm{IUC}(0-1)\} / 3\end{array}\end{array}$ & $\begin{array}{l}\text { TUE = Indicador de Taxa de } \\
\text { Urbanizaçăo do Entorno da } \\
\text { sub-bacia; IAPP = Indicador } \\
\text { de Integridade de Área de } \\
\text { Preservaçäo Permanente; } \\
\text { CUC = Indicador de cobertura } \\
\text { por unidade de Conservação }\end{array}$ \\
\hline
\end{tabular}

Fonte: ISAIAS (2008)

Figura 2 - Fluxograma apresentando as dimensões, indicadores e variáveis básicas que compõe o ISBH

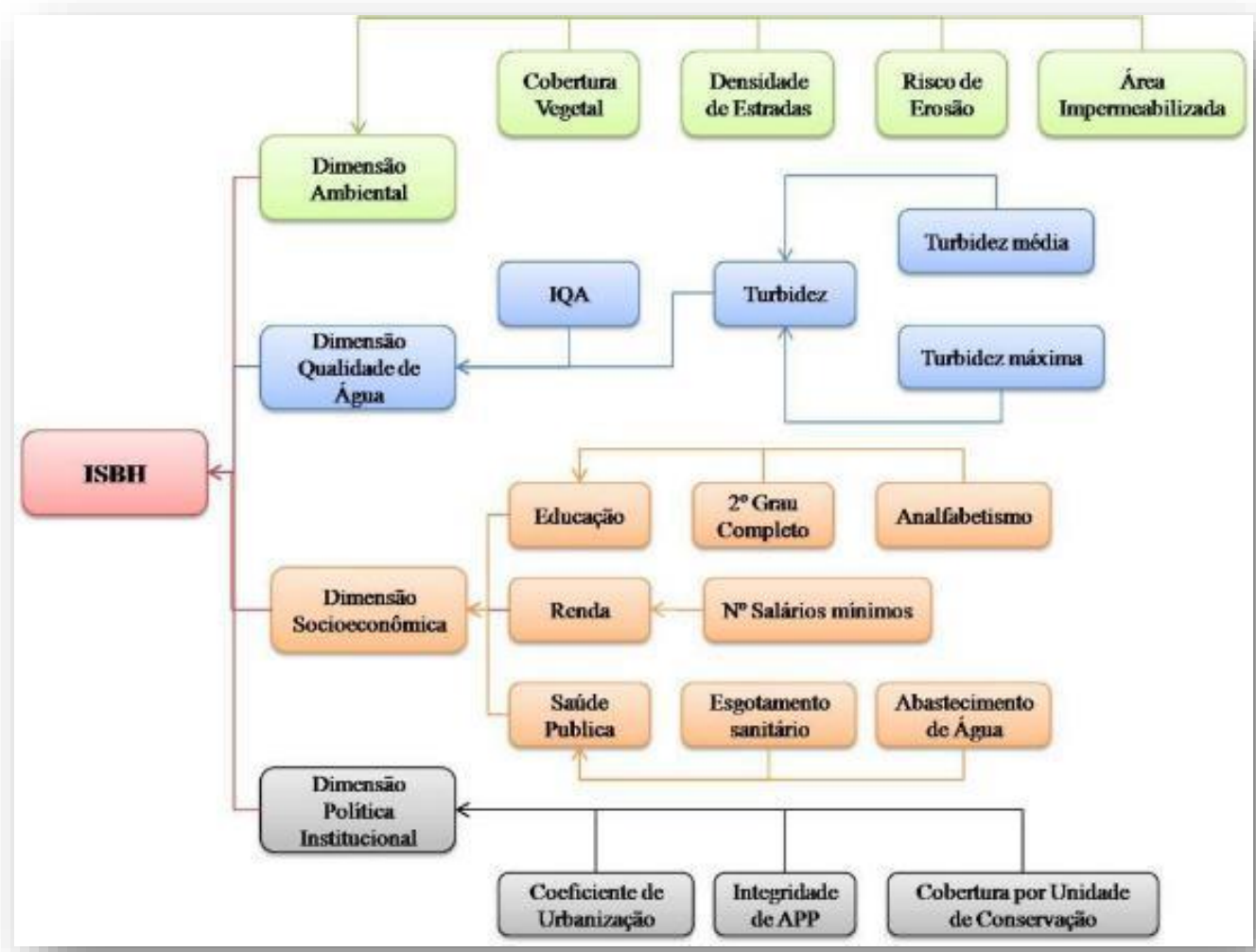


A composição e metodologias para cálculo dos componentes do ISBH são apresentadas no Quadro 1.

Quadro 1 - Resumo metodológico do cálculo dos indicadores que compõem as dimensões do ISBH

\begin{tabular}{|c|c|c|c|c|c|c|}
\hline Dimensão & $\begin{array}{l}\text { Unidade- } \\
\text { Indicador }\end{array}$ & Metodologia para mensuração & & Pon & tuação & \\
\hline \multirow{26}{*}{$\begin{array}{l}\text { Dimensão } \\
\text { Ambiental }\end{array}$} & \multirow{6}{*}{$\begin{array}{l}\quad \% \\
\text { Cobertura } \\
\text { Vegetal/ }\end{array}$} & \multirow{6}{*}{$\begin{array}{c}\text { Obtido a partir do mapeamento de } \\
\text { series históricas da \% de Cobertura } \\
\text { Vegetal (CV) por tipologia nas bacias } \\
\text { estudadas }\end{array}$} & $\mathrm{CV}>90$ & 1 & \multirow{6}{*}{-} & \multirow{6}{*}{-} \\
\hline & & & $90 \geq \mathrm{CV}>80$ & 0.8 & & \\
\hline & & & $80 \geq C V>70$ & 0.6 & & \\
\hline & & & $70 \geq C V>60$ & 0.4 & & \\
\hline & & & $60 \geq C V>50$ & \begin{tabular}{|l|}
0.2 \\
\end{tabular} & & \\
\hline & & & CV $\leq 50$ & 0 & & \\
\hline & \multirow{6}{*}{$\begin{array}{l}\text { ton/ha/ano } \\
\text { Risco de } \\
\text { Erosão/ }\end{array}$} & \multirow{6}{*}{$\begin{array}{l}\text { O indicador reflete a \% da bacia com } \\
\text { Perda de Solo nas classes: } \\
\text { moderada a forte; forte; muito forte; } \\
\text { ou extrema (Ribeiro e Alves, } 2007 \text { ). } \\
\text { para a série histórica que será } \\
\text { utilizada. O cálculo será realizado por } \\
\text { aplicação da Equaçäo Universal de } \\
\text { Perda dos Solos. A equaçäo agrega } \\
\text { os fatores de: erosividade da chuva, } \\
\text { em MJ.mm/(ha.ano); erodibilidade } \\
\text { do solo, em t.h/(MJ.mm); } \\
\text { comprimento de rampa e inclinaçäo } \\
\text { (declividade) da encosta em metros e } \\
\text { \%, respectivamente; e de uso, } \\
\text { manejo e práticas conservacionistas } \\
\text { do solo (adimensional) na área. }\end{array}$} & $P S>12$ & 1 & \multirow{6}{*}{ - } & \multirow{6}{*}{-} \\
\hline & & & $12 \geq P S>9$ & 0.8 & & \\
\hline & & & $9 \geq P S>6$ & 0.6 & & \\
\hline & & & $6 \geq P S>4$ & 0.4 & & \\
\hline & & & $4 \geq P S>2$ & 0.2 & & \\
\hline & & & $P S \geq 2$ & 0 & & \\
\hline & \multirow{7}{*}{$\begin{array}{l}\mathrm{Km} \\
\text { Densidade } \\
\text { de } \\
\text { Estradas }\end{array}$} & \multirow{7}{*}{$\begin{array}{c}\text { Para o cálculo de Densidade } \\
\text { Estradas (DE) será extraída a razão } \\
\text { entre o comprimento total das } \\
\text { estradas de terra e asfalto dentro de } \\
\text { cada bacia em Km e a área de cada } \\
\text { bacia em } \mathrm{Km}^{2} \text {. O indicador será } \\
\text { calculado para toda a histórica que } \\
\text { será analisada }\end{array}$} & $\mathrm{De}<2$ & 1 & \multirow{7}{*}{-} & \multirow{7}{*}{ - } \\
\hline & & & $2<\operatorname{de} \leqslant 2.5$ & 0.8 & & \\
\hline & & & $2.5<$ des 3 & 0.6 & & \\
\hline & & & $3<$ de $\leq 4$ & 0.4 & & \\
\hline & & & $4<$ des 5 & 0.2 & & \\
\hline & & & De $>5$ & 0 & & \\
\hline & & & Als5 & 1 & & \\
\hline & \multirow{7}{*}{$\begin{array}{c}\% \\
\text { Área } \\
\text { Impermea- } \\
\text { bilizada }\end{array}$} & \multirow{7}{*}{$\begin{array}{l}\text { Para o cálculo de Densidade } \\
\text { Estradas será extraída a razão entre } \\
\text { o comprimento total das estradas de } \\
\text { terra e asfalto dentro de cada bacia } \\
\text { em Km e a área de cada bacia em } \\
\mathrm{Km}^{2} \text {. O indicador será calculado para } \\
\text { toda a histórica que será analisada }\end{array}$} & $5<\mathrm{A} \mid \leq 8$ & 0.8 & \multirow{7}{*}{-} & \multirow{7}{*}{-} \\
\hline & & & $8<\mathrm{A} \mid \leq 12$ & 0.6 & & \\
\hline & & & $12<\mathrm{A} \mid \leq 15$ & \begin{tabular}{|l|}
0.4 \\
\end{tabular} & & \\
\hline & & & $15<\mathrm{A} \mid \leq 20$ & 0.2 & & \\
\hline & & & $A l>20$ & 0 & & \\
\hline & & & $\mathrm{De}<2$ & 1 & & \\
\hline & & & $2<$ des 2.5 & 0.8 & & \\
\hline \multirow{12}{*}{$\begin{array}{c}\text { Dimensão } \\
\text { Qualidade de } \\
\text { Agua }\end{array}$} & \multirow{6}{*}{$\begin{array}{l}\text { Classifica- } \\
\text { ção } \\
\text { CETESB } \\
\text { Índice de } \\
\text { Qualidade } \\
\text { de Água }\end{array}$} & \multirow{6}{*}{$\begin{array}{l}\text { Mensurado pela aplicação do Índice } \\
\text { de Qualidade de Agua da CETESB } \\
\text { em pontos de amostragem definidos } \\
\text { posteriormente. As variáveis que o } \\
\text { compöem säo: oxigênio dissolvido, } \\
\text { coliformes termotolerantes, pH. } \\
\text { demanda bioquímica de oxigênio, } \\
\text { temperatura, nitrogênio total, fósforo } \\
\text { total, turbidez e resíduo total }\end{array}$} & Tbms1 & 1 & Tbmx $\leq 10$ & 1 \\
\hline & & & $1<$ Tbms2 & 0.8 & $10<$ Tbmx $\leq 25$ & 0.8 \\
\hline & & & $2<$ Tbms $\leq 3$ & 0.6 & $25<$ Tbmxs50 & 0.6 \\
\hline & & & $3<$ Tbm $\leq 4$ & 0.4 & $50<$ Tbm $x \leq 75$ & 0.4 \\
\hline & & & $5<$ Tbms6 & 0.2 & $75<$ Tbm $x \leq 100$ & 0.2 \\
\hline & & & $T b m>5$ & 0 & Tbm $x>100$ & 0 \\
\hline & \multirow{6}{*}{$\begin{array}{c}\text { NTU } \\
\text { Turbidez }\end{array}$} & & $R>3$ & 1 & \multirow[t]{6}{*}{ 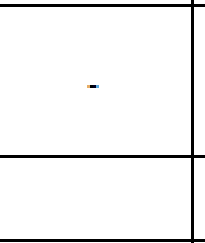 } & \multirow{6}{*}{-} \\
\hline & & turbidez dos corpos hidricos & $3>\mathrm{R}<2,5$ & 0.8 & & \\
\hline & & ino & $2,5 \leq R<2$ & 0.6 & & \\
\hline & & dia & $2 \leq R<1,5$ & 0.4 & & \\
\hline & & Turbidez Média e a variável básica & $1,5 \leq R<0,75$ & 0.2 & & \\
\hline & & $\begin{array}{c}\text { Turbidez Máxima } \\
\end{array}$ & $\mathrm{R} \leq 0,75$ & 0 & & \\
\hline & & Realizaçäo de levantamentos com & $R>3$ & 1 & & \\
\hline & & aplicação de questionários e & $3>\mathrm{R}<2,5$ & 0.8 & & \\
\hline $\begin{array}{l}\text { Dimensao } \\
\text { Socioeco- }\end{array}$ & $\begin{array}{l}\text { Salano } \\
\text { Mínimo }\end{array}$ & levantamento de dados secundários. & $2,5 \leq R<2$ & 0.6 & & \\
\hline nômica & Renda & O indicador de renda será a renda & $2 S R<1.5$ & 0.4 & - & \\
\hline & & per capta das localidades inseridas & $1,5 \leq \mathrm{R}<0,75$ & 0.2 & & \\
\hline & & & $\mathrm{R} \leq 0,75$ & 0 & & \\
\hline
\end{tabular}




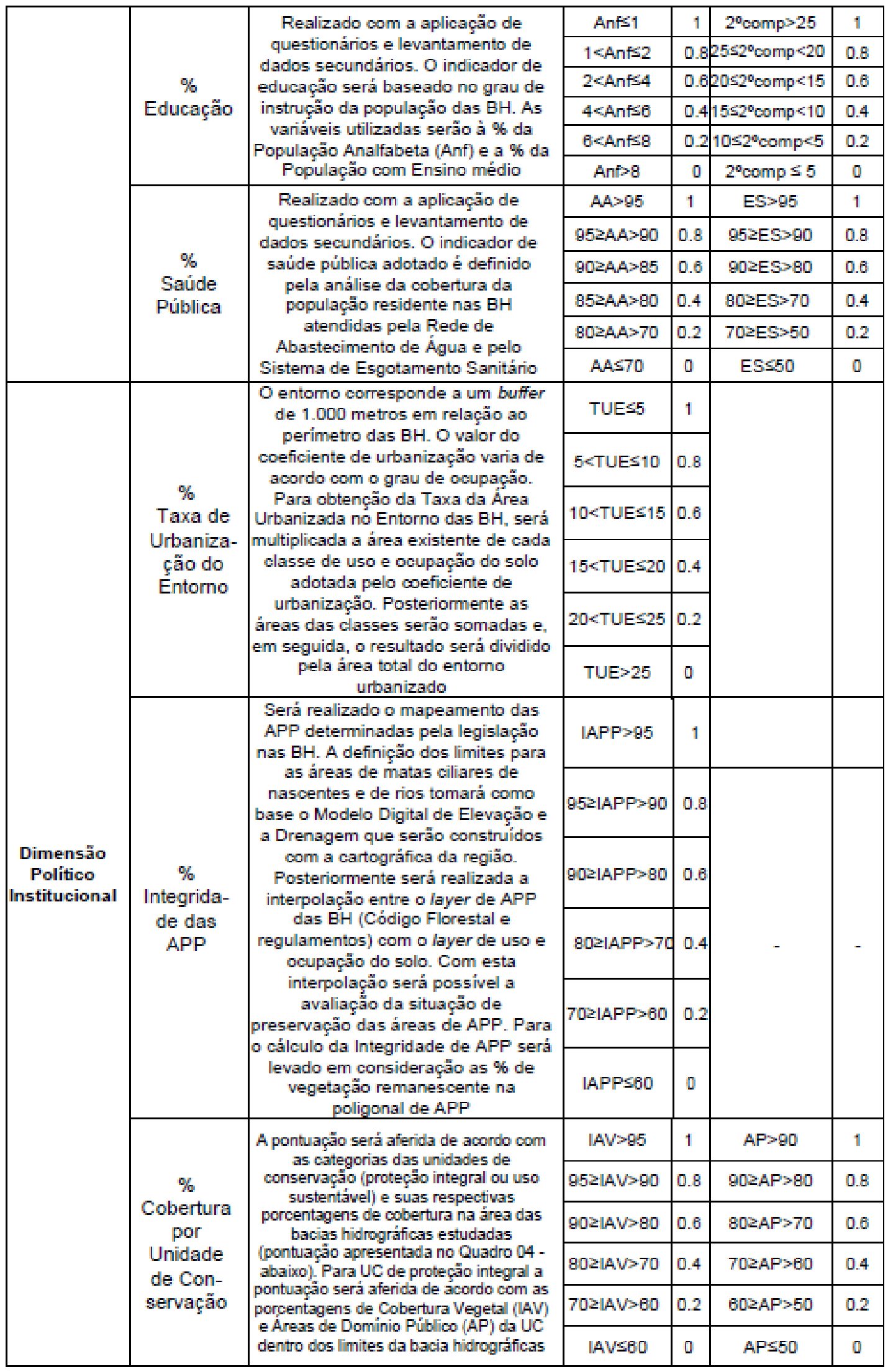

Fonte: Autores (2018). 


\section{AS DIMENSÕES AMBIENTAL E SOCIOECONÔMICA DAS SBH DO BA- TATÃ E MARACANÃ}

De acordo com o mapeamento realizado para uso e ocupação do solo, foram calculadas as áreas de vegetação (capoeira alta, média, baixa e manguezal) para as SBH estudadas no ano de 2008. Nas SBH do Batatã e Maracanã, o percentual de vegetação foi de 77,9\% na primeira e de 45,7\% na segunda, respectivamente. As pontuações do ISBH para este foram de 0,6 no Batatã e 0 no Maracanã.

Com relação a perda de solos, a classe predominante para ambas as SBH foram muito baixas, ou seja, com perda de solo entre 0 e 1 tonelada/hectare/ano (Tabela 2). Com relação à pontuação do indicador para as SBH os valores foram de 0,8 para o Batatã e 0,4 para o Maracanã.

Tabela 2 - Classe de Perda de Erosão para as SBH do Batatã e Maracanã

\begin{tabular}{c|c|c|c|c}
\hline \multirow{2}{*}{ Classes de Perda de Solo (ton/h.ano) } & \multicolumn{2}{c}{ Sub-bacia do Batatã } & \multicolumn{2}{c}{ Sub-bacia do Maracanã } \\
\cline { 2 - 5 } & \multicolumn{2}{|c}{$\mathbf{2 0 0 8}$} & \multicolumn{2}{c}{$\mathbf{2 0 0 8}$} \\
\cline { 2 - 5 } & Área (ha) & $\%$ & Área (ha) & $\%$ \\
\hline Muito baixa & $1.284,90$ & 74,19 & $1,478,70$ & 54,77 \\
\hline Baixa & 213,05 & 12,30 & 396,16 & 14,67 \\
\hline Baixa a moderada & 183,87 & 10,62 & 460,74 & 17,06 \\
\hline Moderada & 7,64 & 0,44 & 145,69 & 5,40 \\
\hline Moderada a forte & 37,69 & 2,18 & 189,77 & 7,03 \\
\hline Forte & 3,41 & 0,20 & 20,27 & 0,75 \\
\hline Muito Forte & 1,38 & 0,08 & 8,58 & 0,32 \\
\hline Extrema & 0 & 0 & 0,0004 & 0,00001 \\
\hline
\end{tabular}

Fonte: Autores (2018).

Para o indicador densidade de estradas, foram mapeados 52,75 Km e 17,56 Km de estradas de terra e asfalto para a SBH do Batatã, respectivamente. Com relação ao Maracanã os valores obtidos foram de 101,2 $\mathrm{Km}$ de estradas de terra e 73,59 $\mathrm{Km}$ de asfalto. Os resultados de densidade de estrada foram de 4,57 $\mathrm{Km}^{-1}$ para a SBH do Batatã equivalendo a 0,2 pontos para o indicador e de $6,47 \mathrm{Km}^{-1}$ para a SBH do Maracanã, correspondendo a pontuação 0 . O indicador áreas impermeáveis quantificou que as SBH do Batatã e Maracanã possuíam 11,37\% e $66 \%$ de áreas impermeáveis na sua superfície. A pontuação obtida para o indicador foi de 0,6 para o Batatã e 0 para o Maracanã.

A Figura 3 apresenta os valores calculados para o Índice de Qualidade da Água e as respectivas classificações obtidos para as SBH do Batatã e Maracanã nos períodos de estiagem e chuvoso. Para a pontuação do indicador foi extraído a média dos períodos de estiagem e chuvoso dos pontos de amostragem para cada SBH. Os valores médios foram de 68,86 para a SBH do Batatã e 65,26 para a SBH do Maracanã, perfazendo 0,4 pontos para o indicador em ambas $\mathrm{SBH}$. 
Figura 3 - Pontuação do Índice de Qualidade da Água das SBH do Batatã e Maracanã para as estações de estiagem e chuva nos seis pontos de amostragem/2009

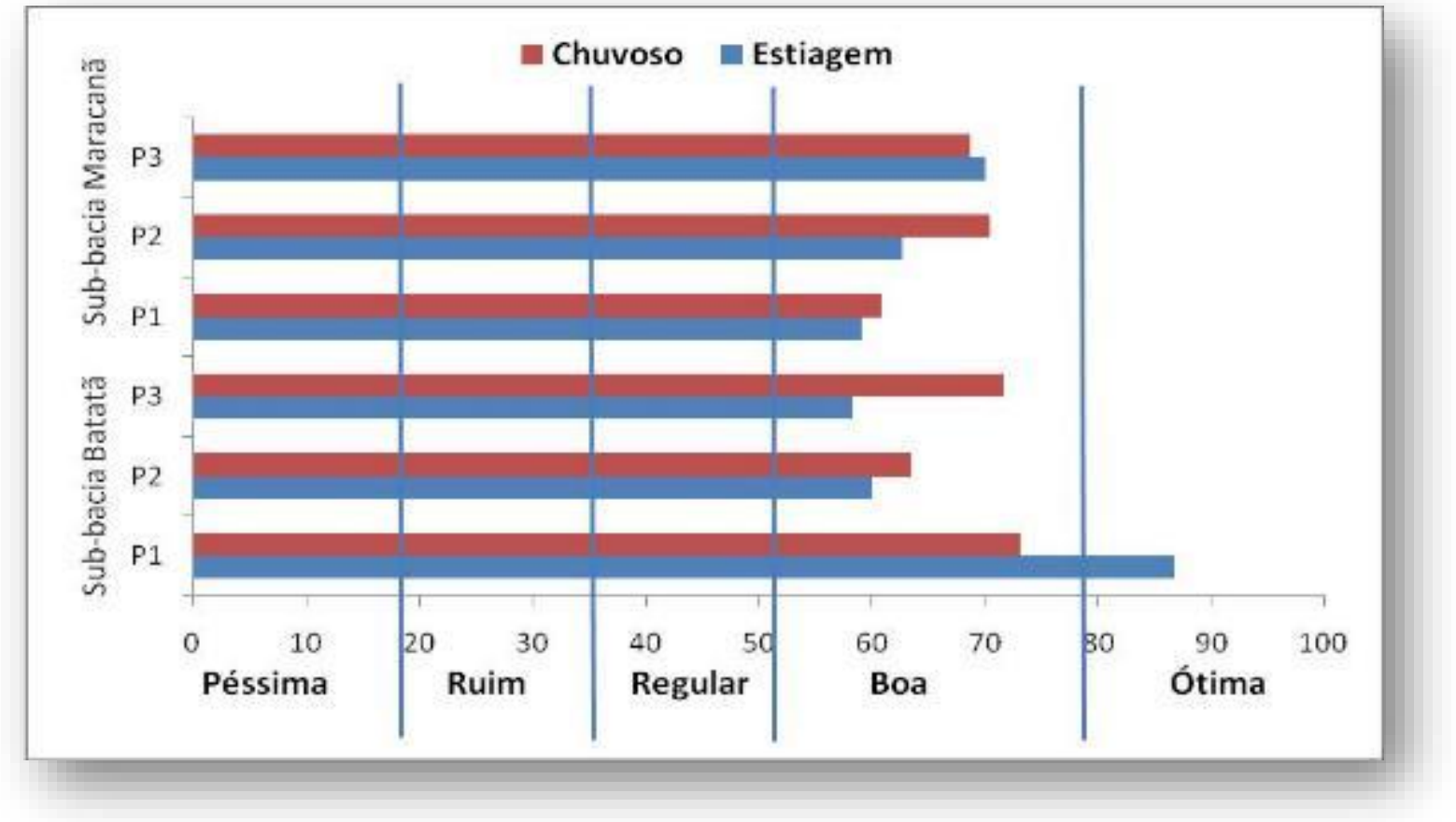

Fonte: Autores (2018).

Para a SBH do Batatã o valor de turbidez média anual foi de 8,03, considerando o Nephelometric Turbidity Unity (NTU) e a máxima de 19,9 (NTU) que corresponderam a pontuação 0 e 0,8 , respectivamente. A pontuação geral para este indicador nesta SBH foi de 0,4 . Na SBH do Maracanã o valor de turbidez média anual foi de 27,94 (NTU) e a máxima de 150 (NTU), atribuindo a pontuação 0 para os dois critérios.

Com relação a escolaridade no Batatã, apenas 34\% da população possuem o ensino médio completo, cerca de 47,54\% tem a escolaridade entre o ensino fundamental incompleto e oitava série. Quanto às porcentagens de pessoas cursando ou com $3^{\circ}$ grau concluído apenas 2,4\% estão enquadradas nestas duas classes. No Maracanã, a maioria da população concluiu o ensino médio (48\%), 33,22\% pessoas cursaram entre o ensino fundamental e a oitava série, a porcentagem de analfabetismo é de 5,7\%. A pontuação do indicador foi de 0,8 para o Batatã e de 0,7 para o Maracanã.

Para o indicador renda, a interpolação dos questionários mostrou que a renda per capita das SBH estudadas foi de R \$280,00 e R \$310,00 para do Batatã e Maracanã, respectivamente. Com estes rendimentos a pontuação deste indicador foi de 0 para as duas SBH. A pontuação do indicador renda per capita refletiu a realidade socioeconômica dos bairros e comunidades inseridos nas $\mathrm{SBH}$ estudadas. Em ambas a renda per capita mostrou-se inferior a 1 salário mínimo, o que provavelmente influencia diretamente na qualidade ambiental da região, pois muitas famílias precisam extrair recursos naturais remanescentes na região para sua subsistência.

Os levantamentos em campo mostraram que na $\mathrm{SBH}$ Batatã 87,5\% das residências são atendidas pelo sistema de abastecimento de água, no entanto apenas 8,33\% possuem interligação com a rede de esgotos. Para a SBH do Maracanã 46,29\% possuem interligação com a rede de abastecimento de água e 3\% possuem rede de coleta de esgotos.

As pontuações deste indicador demonstraram a precariedade dos serviços de esgotamento sanitário e abastecimento de água das SBH estudadas. A SBH do Batatã obteve a pontuação 0,3 devido os serviços que são oferecidos pela Companhia de Saneamento Ambiental do Maranhão (CAEMA) nos bairros Vila Itamar, Vila Sarney e Vila Esperança. No Maracanã a situação é mais crítica, pontuação igual a 0. De acordo 
com os questionários aproximadamente 50\% das residências utilizam-se de águas de poços próprios e de vizinhos para suas necessidades básicas.

Com relação ao indicador taxa de urbanização do entorno, o percentual calculado para a SBH do Batatã foi de 21,46\%, totalizando 0,4 pontos. Na SBH do Maracanã, o valor foi 16,74\%, correspondendo a 0,8 pontos. Para o indicador Integridade das Áreas de Preservação Permanente (APP), a delimitação mostrou que 474,94 ha e 525,62 hectares que correspondem a 27,43\% e 19,41\% das áreas das SBH do Batatã e Maracanã, respectivamente, são APP. A pontuação do indicador foi de 0,4 para o Batatã e Maracanã. A Figura 4 ilustra os diferentes tipos de uso e cobertura da terra na poligonal das APP.

Figura 4 - Integridade das APP para as SBH do Batatã e Maracanã referentes/2008

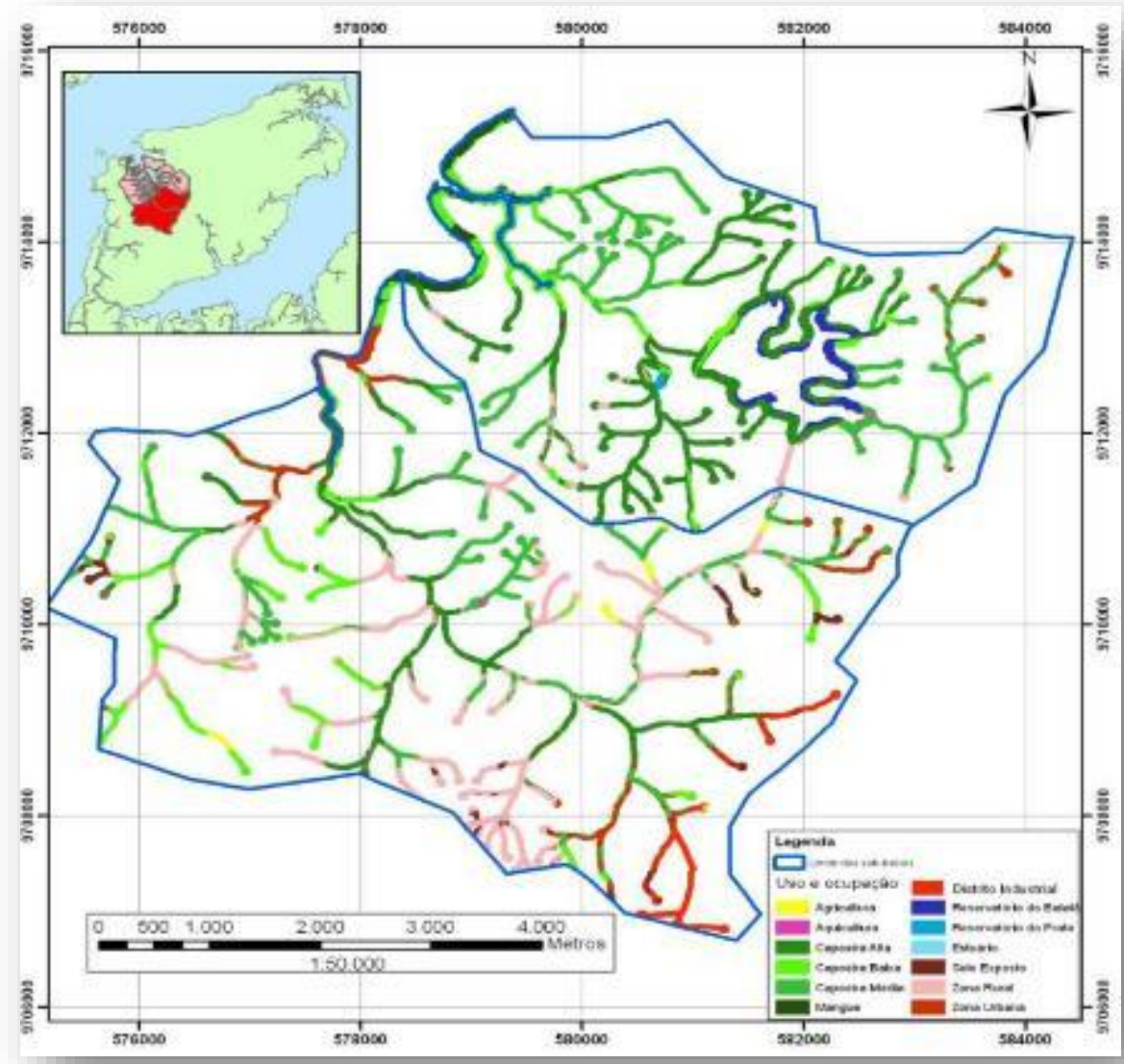

Fonte: Autores (2018).

Sobre o Indicador de Unidades de Conservação (UC), na área de estudo se sobrepõem aos limites de três UC e uma Zona de Reserva Florestal, sendo elas: Parque Estadual do Bacanga - UC de Proteção Integral, criada pelo Decreto Lei Estadual no 7545/1980; Área de Proteção Ambiental do Maracanã - UC de Uso Sustentável, criada pelo Decreto Estadual no 12.103/91; Área de Proteção Ambiental Upaon-açu Miritiba/Alto Preguiça - UC de Uso Sustentável, criada pelo Decreto Estadual no 12428/1992; e a Zona de Reserva Florestal do Sacavém, que apesar de não ser considerada como UC possui restrições quanto ao seu uso, sendo criada pala Lei Municipal no 3.253/92.

De acordo com os resultados do mapeamento das UC, constatou-se que a SBH do Batatã possui 1.356 hectares, correspondente a 78\% de sua área coberta por UC de Proteção Integral (Parque Estadual do Bacanga) e 487,4 ha de cobertura por UC de uso sustentável (Área de Proteção Ambiental do Maracanã), o que corresponde a $12,4 \%$ do seu território. 
Para o Maracanã os valores foram de 214,8 hectares e 1.670 hectares de coberturas por UC de uso sustentável (Área de Proteção Ambiental do Maracanã e Área de Proteção Ambiental Upaon-açú/Miritiba/Alto Preguiça) e proteção integral (Parque Estadual do Bacanga) que corresponderam a 18,05\% e $61,88 \%$ do seu território, respectivamente. As pontuações para o ISBH foram de 1 para o Batatã e 0,4 para o Maracanã.

O cálculo da porcentagem de Vegetação da UC levou em consideração o somatório das áreas de capoeira alta, média, baixa e mangue que ocorrem no limite do Parque Estadual do Bacanga em relação às SBH. A pontuação para as UC de Proteção Integral foi realizada por meio da extração da média da pontuação atribuída para as porcentagens de Vegetação e Áreas Pública do Parque Estadual do Bacanga, inserido em cada SBH estudada. Sendo assim, foi conferido o valor de 0,7 pontos para a SBH do Batatã e 0,2 pontos para o Maracanã.

Com relação ao Indicador UC, a pontuação levou em consideração a média entre os critérios de Cobertura por UC de uso sustentável e UC de proteção integral. Os valores foram de 0,8 para o Batatã e de 0,3 para o Maracanã.

\section{ANÁLISE INTEGRADA DO ÍNDICE DE SUSTENTABILIDADE DE BACIAS HIDROGRÁFICAS}

A pontuação obtida para a dimensão ambiental foi de 0,55 e 0,15 para as SBH do Batatã e Maracanã, respectivamente. Para a dimensão de qualidade de água, a pontuação foi de 0,4 e 0,2 para as SBH do Batatã e Maracanã. A pontuação da dimensão socioeconômica foi de 0,37 e 0,23 as SBH do Batatã e Maracanã, respectivamente. A dimensão político-institucional foi pontuada com 0,55 e 0,37 pontos para as $\mathrm{SBH}$ do Batatã e Maracanã, respectivamente.

Quando ao ISBH, os resultados obtidos foram de 0,47 e 0,24 pontos para as SBH do Batatã e Maracanã, respectivamente. Dentre as dimensões a que apresentou maiores valores variações entre estas duas SBH foi a ambiental, seguida pela de qualidade de água, político-institucional e socioeconômica, respectivamente. A figura 5, apresenta os valores do ISBH com as respectivas pontuações das dimensões que o compõem para as SBH estudadas. A Tabela 3 apresenta resumo integrado de todas as pontuações dos indicadores, dimensões que compõem o ISBH e do próprio ISBH. 
Figura 5 - Resultados do ISBH, dimensões ambientais, qualidade de água, socioeconômica e político-institucional - SBH do Batatã e Maracanã

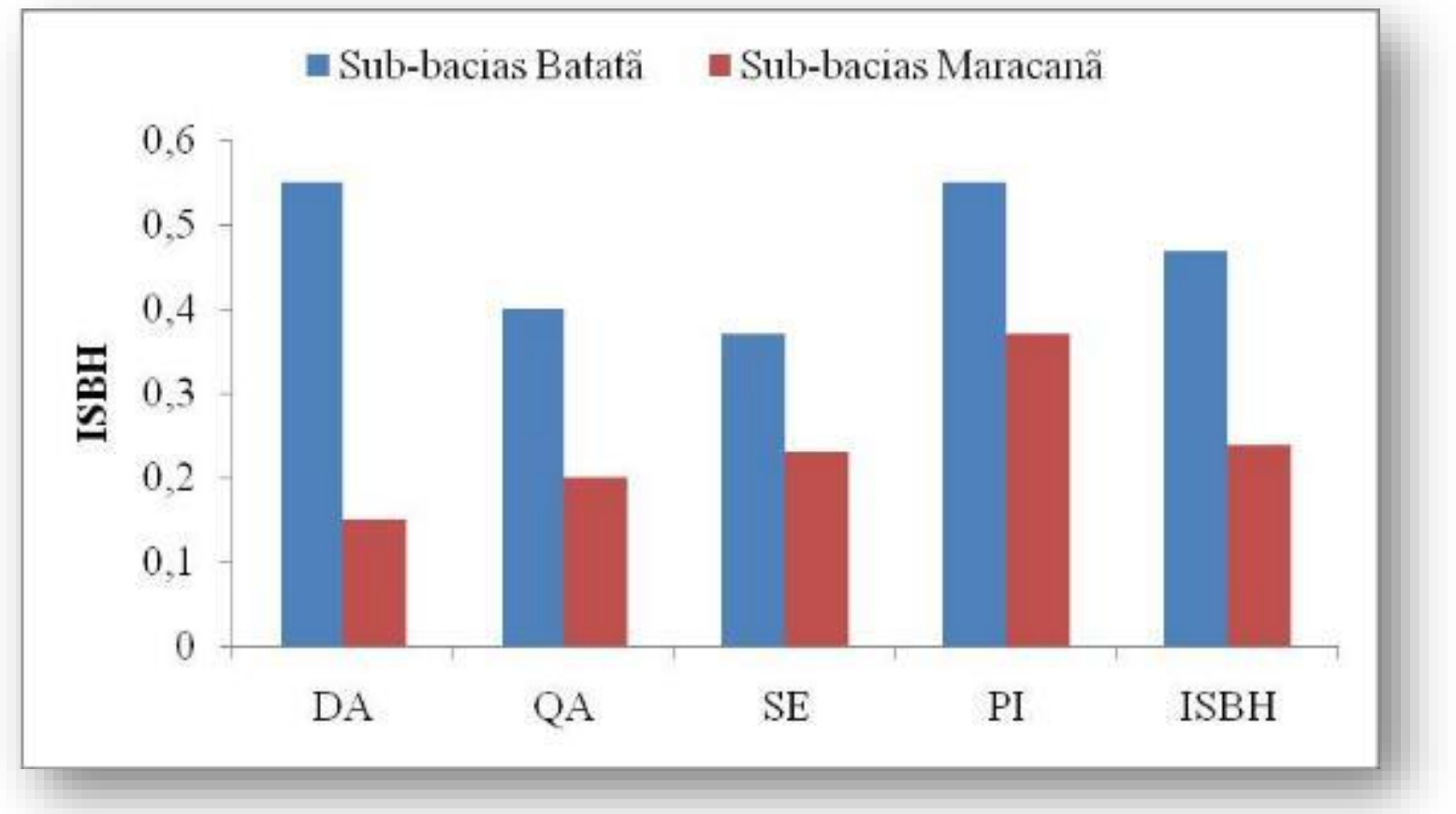

Fonte: Autores (2018).

Tabela 3 - Resultados dos indicadores e dimensões que compõem o ISBH e o próprio ISBH para as SBH do Batatã e Maracanã

\begin{tabular}{|c|c|c|}
\hline Indicadores/Dimensões & SBH Batatã & SBH Maracanã \\
\hline Cobertura Vegetal & 0,6 & 0 \\
\hline Risco de Erosão & 0,8 & 0,4 \\
\hline Densidade de Estradas & 0,2 & 0,2 \\
\hline Área Impermeabilizada & 0,6 & 0 \\
\hline Dimensão Ambiental & 0,55 & 0,15 \\
\hline IQA & 0,4 & 0,4 \\
\hline Turbidez & 0,4 & 0 \\
\hline Dimesão Qualidade de Água & 0,4 & 0,2 \\
\hline Educação & 0,8 & 0,7 \\
\hline Renda & 0 & 0 \\
\hline Saúde Pública & 0,3 & 0 \\
\hline Dimensão Socioeconômica & 0,37 & 0,23 \\
\hline Urbanização do entorno & 0,4 & 0,8 \\
\hline Integridade de APP & 0,4 & 0 \\
\hline $\mathrm{UC}$ & 0,85 & 0,3 \\
\hline Dimensão Político-Institucional & 0,55 & 0,37 \\
\hline ISBH & 0,47 & 0,24 \\
\hline
\end{tabular}

Fonte: Autores (2018).

De forma geral, pode-se verificar que a pontuação do ISBH, assim como de seus indicadores retrataram as diferenças e semelhanças entre as SBH estudadas, mostrando que a ferramenta utilizada permite uma análise comparativa entre diferentes áreas. Para que ISBH seja utilizado como ferramenta para a gestão pública de modo a estabelecer critérios de conservação de bacias ou SBH da Ilha do Maranhão é necessário que sejam feitas as adaptações sugeridas neste trabalho. 


\section{CONSIDERAÇÕES FINAIS}

Os objetivos desta pesquisa foram alcançados de forma satisfatória tendo em vista que a metodologia empregada para a aplicação e avaliação do ISBH apresentou resultados coerentes com a realidade da área de estudo, ratificando inclusive observações de campo. O ISBH em seu conjunto e com as propostas de adaptações pode ser utilizado para diagnósticos e comparações das condições de diferentes SBH;

O ISBH poderá ser aproveitado pelo poder público a fim de subsidiar o estabelecimento de prioridades nas tomadas de decisões referentes à elaboração e implementação de planos, programas e políticas que visem à gestão de SBH hidrográficas. A aplicação das técnicas integradas de geoprocessamento foram fundamentais no desenvolvimento do trabalho e se mostraram totalmente eficientes tanto na manipulação e armazenagem dos dados como nas análises realizadas, mostrando-se totalmente adequadas como instrumental de apoio a metodologia utilizada.

\section{REFERÊNCIAS}

CASTRO, T. C. S. Identificação de áreas potenciais para a recarga de aquífero na bacia hidrográfica do rio Bacanga. São Luís. 56p. Monografia (Curso Ciências Aquáticas). Departamento de Oceanografia e Limnologia, Universidade Federal do Maranhão. 2008.

COELHO, C. J. C. Aspectos da Disponibilidade e dos Usos da Água na Bacia do Bacanga/Ilha do Maranhão (Ilha de São Luis)-MA. São Luís, 125 p. Monografia (Curso Ciências Aquáticas). Departamento de Oceanografia e Limnologia, Universidade Federal do Maranhão. 2006.

ISAIAS, F. B. A Sustentabilidade da água: proposta de um índice de sustentabilidade de bacias hidrográficas. Brasília. 156 f. Dissertação de Mestrado. Centro de Desenvolvimento Sustentável, Universidade de Brasília. 2008.

MARANHÃO. Decreto Estadual n 19.145/2002. Dispõe sobre a Gestão do Parque Ecológico Estadual da Lagoa da Jansen, criado pela Lei $N^{\circ}$ 4.878/1988 e dá outras providências. 2002.

PEREIRA, E. D. Avaliação da Vulnerabilidade Natural à Contaminação do Solo e Aquífero do Reservatório Batatã - São Luís (MA). Tese de Doutorado. Rio Claro - SP: UNESP. 2006.

ZEE-MA. Zoneamento Ecológico-Econômico do Estado do Maranhão. Cartas DSG (Diretoria de Serviços Geográficos). 2003. Disponível em: http://www.zee.ma.gov.br/ Acesso em: 28 de jul. de 2009. 